\title{
Supplement
}

\section{Deposition lifetime}

The exchange flux $F$ of various gases between an air and water interface can be phenomenologically be described through $[3,4]$ :

$$
F=K_{G}\left(G-\frac{A}{H}\right) \text {. }
$$

Where $K_{G}$ is the overall mass-transfer coefficient, $\mathrm{G}$ is the gas phase concentration, A the aqueous phase concentration and H the Henry's law constant. If we assume that the aqueous phase concentration is zero (e.g. outside of the Somalia upwelling when travelling towards the ship) then equation 1 simplifies to:

$$
F=K_{G} G .
$$

The mass-transfer coefficient $K_{G}$ can be expressed as:

$$
\frac{1}{K_{G}}=\frac{1}{k_{G}}+\frac{1}{\frac{1}{4} \nu \alpha}+\frac{1}{H k_{L} \beta} .
$$

In this equation $k_{G}$ is the gas phase and $k_{L}$ the liquid phase mass-transfer coefficient, $\nu$ the mean molecular speed of the molecule, $\alpha$ the mass-accommodation coefficient and $\beta$ an enhancement coefficient of the aqueous phase mass transfer flux due to removal of the molecule by chemical reactions. No enhancement means $\beta=1$. The middle term which describes the interfacial resistance can be generally neglected in the natural environment because $\alpha$ is always sufficiently large [3]. If the molecule under investigation has a solubility of $H \gg H_{c r i t}=\frac{k_{G}}{k_{L}}$ then gas phase mass transport is dominant and the flux is independent of $H$. We determined a Henry's law constant in the range of $3.3 \times 10^{4} \mathrm{M} \mathrm{atm}^{-1}-6.5 \times 10^{5} \mathrm{M} \mathrm{atm}^{-1}$ for methane sulfonamide (MSAM) (see Sect. S2) and for $\mathrm{DMSO}_{2}$ literature indicates a value greater than $5 \times 10^{4} \mathrm{M} \mathrm{atm}^{-1}$ [1]. This means that both are sufficiently soluble substances for which the assumption holds true that gas phase mass transport is controlling and the overall mass-transfer coefficient is given in the form:

$$
\frac{1}{K_{G}}=\frac{1}{k_{G}} \text {. }
$$


The gas phase mass-transfer coefficient for oceanic applications can be estimated with the help of the wind speed $v[2]$ :

$$
k_{G}=0.0013 v
$$

for an observation height of $10 \mathrm{~m}$ which is the approximate height of observation during the AQABA campaign. The wind speed during the Arabian sea part in the second leg varied around $4 \mathrm{~m} \mathrm{~s}^{-1}$ and $14 \mathrm{~m} \mathrm{~s}^{-1}$ which yields: $K_{G}=0.52 \mathrm{~cm} \mathrm{~s}^{-1}\left(\mathrm{v}=4 \mathrm{~m} \mathrm{~s}^{-1}\right)$ and $K_{G}=1.82 \mathrm{~cm} \mathrm{~s}^{-1}\left(\mathrm{v}=14 \mathrm{~m} \mathrm{~s}^{-1}\right)$.

This in turn gives a lifetime (1/e) of $40 \pm 14$ hours for a wind speed of $\mathrm{v}=4 \mathrm{~m}$ $s^{-1}$ and about $11 \pm 4$ hours for $\mathrm{v}=14 \mathrm{~m} \mathrm{~s}^{-1}$. The average marine boundary layer height used for the lifetime calculation was $750 \pm 250 \mathrm{~m}$.

\section{Henry's law constant measurement}

Artificial seawater was prepared by adding $35 \mathrm{~g} \mathrm{NaCl}$ and $0.5 \mathrm{~g} \mathrm{NaHCO}_{3}$ to a combined volume of $1 \mathrm{~L}$ in MilliQ water. The MSAM mixing ratio of the headspace of $0.05 \mathrm{~mol} \mathrm{~L}^{-1}$ and $0.0005 \mathrm{~mol} \mathrm{~L}^{-1} \mathrm{MSAM}$ in sea water, flushed with $100 \mathrm{ml} \mathrm{min}^{-1}$ of synthetic air (Air Liquide, Krefeld, Germany) each, was measured with a PTR-MS instrument. A range for the Henry's law constant was derived from these measurements as the ratio of the concentration of MSAM in solution (in M) vs. the measured partial pressure in the gas phase (in atm): $3.3 \times 10^{4} \mathrm{M} \mathrm{atm}^{-1}-6.5 \times 10^{5} \mathrm{M} \mathrm{atm}^{-1}$. Measurements were performed at $25^{\circ} \mathrm{C}$ and 995 mbar.

\section{$3 \quad$ Weighting factors}

Chlorophyll a water content encountered during transport of the airmasses towards the ship was weighted according to time before arrival at the ship. We employed a linear and an exponential weighting factor.

\subsection{Linear weighting factors}

The linear weighting factor is in the form $w=\frac{1}{1+p * t}$, where $p$ is the weighting parameter and $t$ is the number of hours before arrival at the ship's location. The chlorophyll a water content is multiplied by the weighting factor $w$ to get the weighted chlorophyll $a$ water content. We varied the $p$ in the range from $p=0.02$ to $p=1$. The resulting plots are displayed in Fig. 1 and Fig. 2. A weighting parameter of $p=0.02$ results in a high contribution and a $p=1$ in a low contribution of chlorophyll $a$ water content further away of 
the ship. Calculations of leg 1 with low weighting parameters $p=0.02-0.1$ lead to a small increase in total chlorophyll a exposure of the trajectories but not in the exposure in the Somalia upwelling compared to other higher weighting parameters. This means that chlorophyll a pick up further away than the Somalia upwelling is responsible for this. MSAM and $\mathrm{DMSO}_{2}$ are low or not detected during leg 1 , therefore chlorophyll exposure in regions further away than the Somalia upwelling does not appear to play a role. The calculations in leg 2 are generally quite similar and therefore independent of the weighting parameter $p$. 


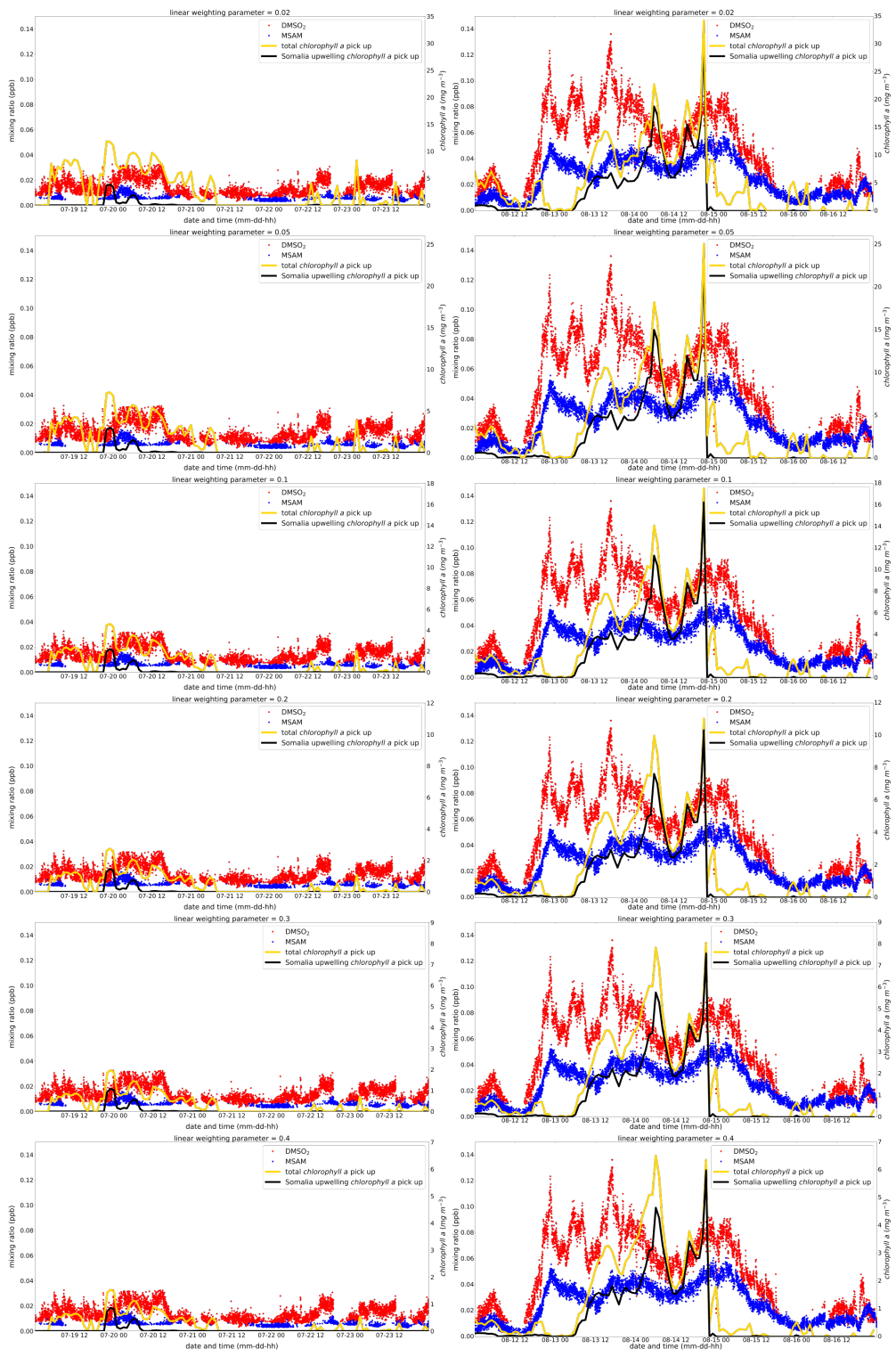

Figure 1: Linear weighting parameters from $p=0.02-0.4$. The total chlorophyll a exposure (yellow line) and the chlorophyll a exposure originating from the Somalia upwelling region (black line) is plotted. The corresponding $\mathrm{y}$-axis for the chlorophyll a exposure is displayed on the right side. Measured ambient mixing ratios in ppb for $\mathrm{DMSO}_{2}$ and MSAM are plotted in red and blue with the corresponding y-axis on the left side. In the left column leg 1 and in the right column leg 2 is displayed. 


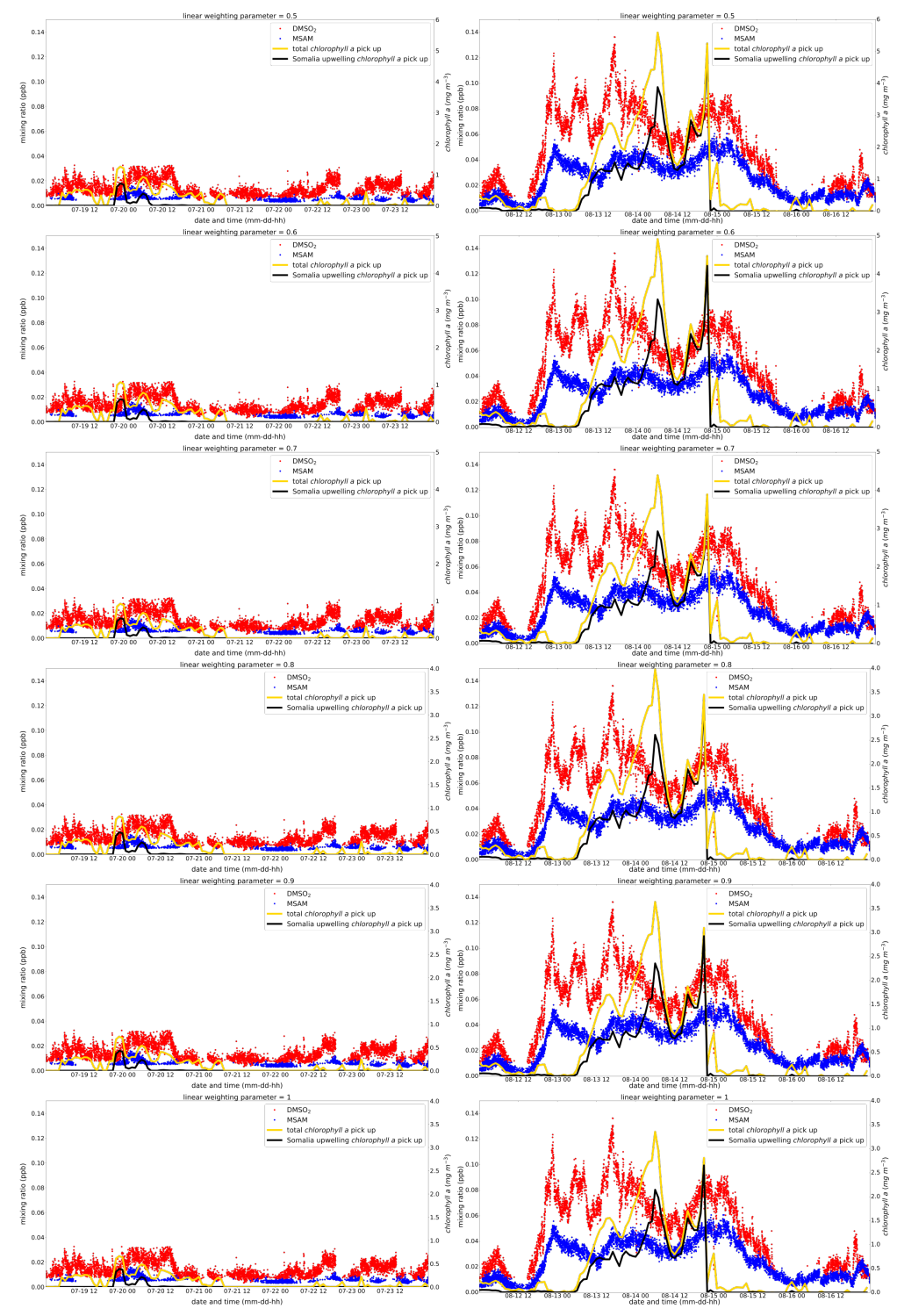

Figure 2: Linear weighting parameters from $p=0.5-1$. The total chlorophyll a exposure (yellow line) and the chlorophyll a exposure originating from the Somalia upwelling region (black line) is plotted. The corresponding $\mathrm{y}$-axis for the chlorophyll a exposure is displayed on the right side. Measured ambient mixing ratios in ppb for $\mathrm{DMSO}_{2}$ and MSAM are plotted in red and blue with the corresponding y-axis on the left side. In the left column leg 1 and in the right column leg 2 is displayed. 


\subsection{Exponential weighting factors}

The exponential weighting factor has the form $w_{\text {exp }}=p^{t}, p$, where $p$ is the weighting parameter and $t$ is the number of hours before arrival at the ship's location. The weighting factor is then multiplied with the respective chlorophyll $a$ water content to yield the weighted chlorophyll $a$. The weighting parameter was varied from $p=0.8$ to $p=0.99$ (see Fig. 3 and Fig. 4). A weighting parameter of 1 means that all chlorophyll a water content is weighted equally. If $p$ is close to 1 we see in the first leg a small increase in total chlorophyll a exposure coming from chlorophyll a pick up further away as the Somalia upwelling (as for the linear case). For the other cases we see in leg 2 that the Somalia upwelling region always constitutes the mayor part of the total chlorophyll a exposure, even in the case of $p=0.8$, which discriminates strongly against chlorophyll exposure further away from the ship. 


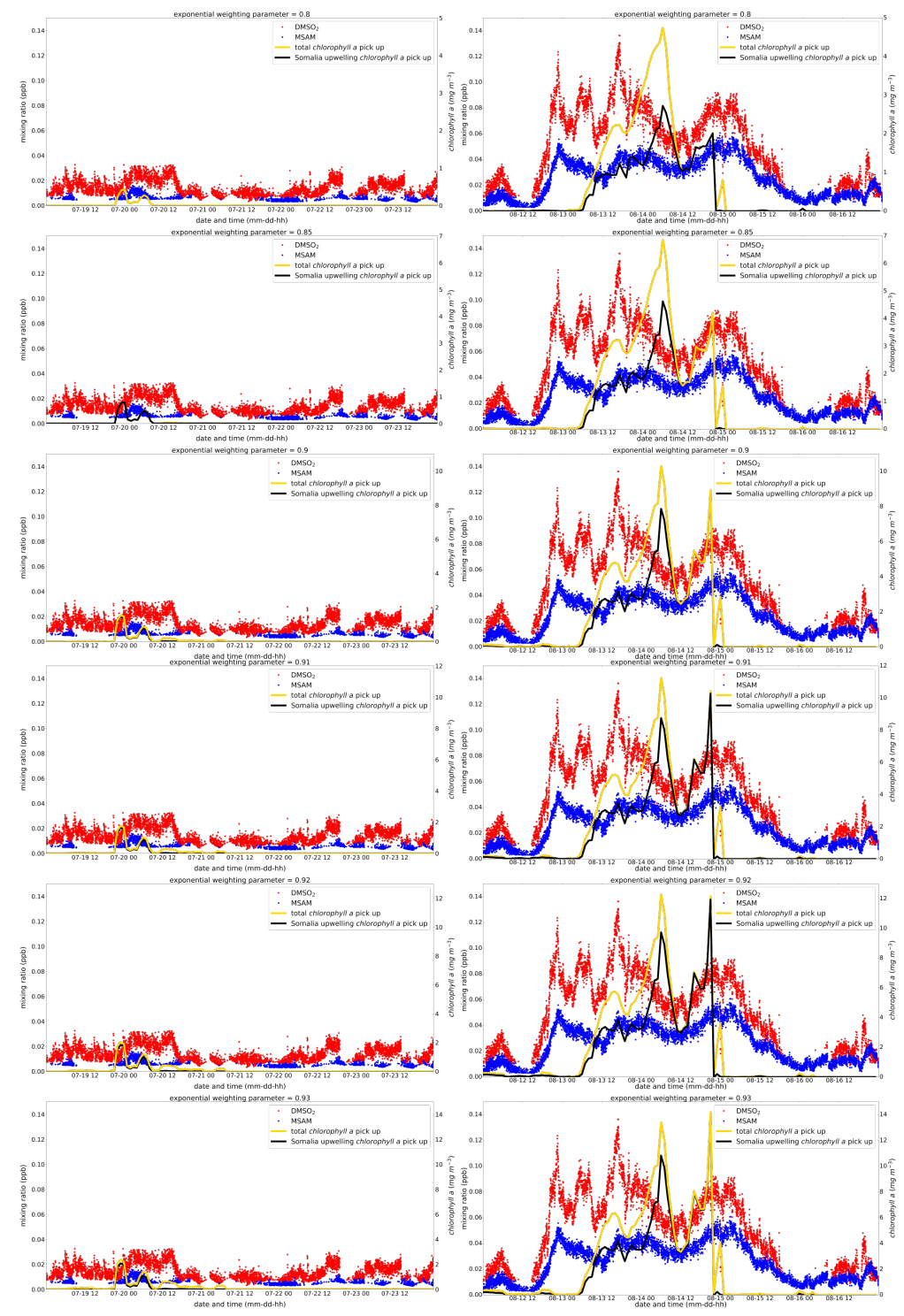

Figure 3: Exponential weighting parameters from $p=0.8-0.93$. The total chlorophyll a exposure (yellow line) and the chlorophyll a exposure originating from the Somalia upwelling region (black line) is plotted. The corresponding y-axis for the chlorophyll a exposure is displayed on the right side. Measured ambient mixing ratios in ppb for $\mathrm{DMSO}_{2}$ and MSAM are plotted in red and blue with the corresponding y-axis on the left side. In the left column leg 1 and in the right column leg 2 is displayed. 


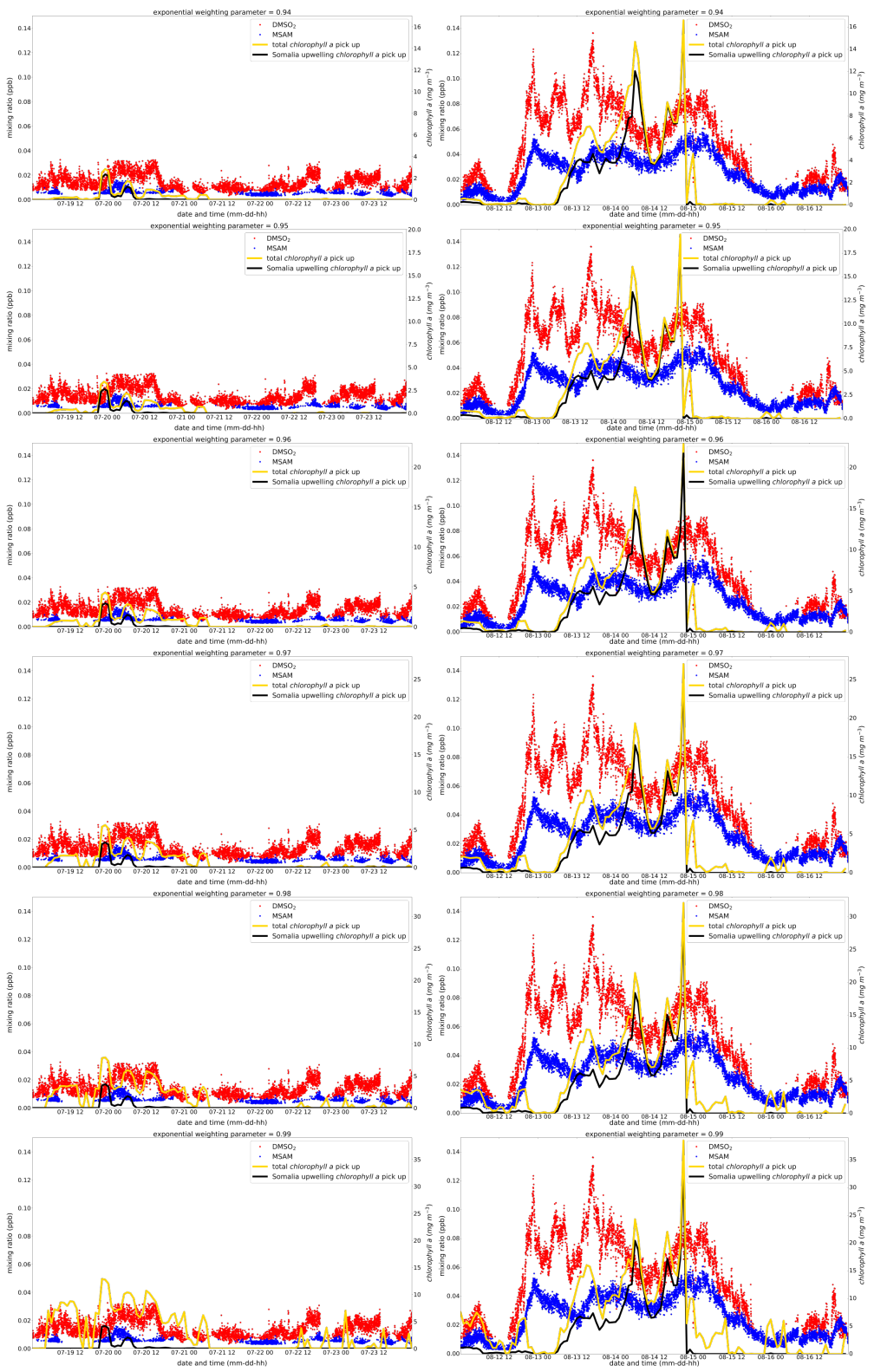

Figure 4: Exponential weighting parameters from $p=0.94-0.99$. The total chlorophyll a exposure (yellow line) and the chlorophyll a exposure originating from the Somalia upwelling region (black line) is plotted. The corresponding $\mathrm{y}$-axis for the chlorophyll a exposure is displayed on the right side. Measured ambient mixing ratios in ppb for $\mathrm{DMSO}_{2}$ and MSAM are plotted in red and blue with the corresponding y-axis on the left side. In the left column leg 1 and in the right column leg 2 is displayed. 


\section{References}

[1] W. J. De Bruyn, Jeffrey A. Shorter, P. Davidovits, D. R. Worsnop, M. S. Zahniser, and C. E. Kolb. Uptake of gas phase sulfur species methanesulfonic acid, dimethylsulfoxide, and dimethyl sulfone by aqueous surfaces. Journal of Geophysical Research: Atmospheres, 99(D8):1692716932, 1994.

[2] B. B. Hicks and P. S. Liss. Transfer of so2 and other reactive gases across the air-sea interface. Tellus, 28(4):348-354, 1976.

[3] S. E. Schwartz. Factors governing dry deposition of gases to surfacewater. In S. E. Schwartz and W. G.N. SLINN, editors, PRECIPITATION SCAVENGING AND ATMOSPHERE-SURFACE EXCHANGE, VOLS 1-3, pages 789-801, NEW YORK, 1992. HEMISPHERE PUBL CORP.

[4] M. Yang, R. Beale, P. Liss, M. Johnson, B. Blomquist, and P. Nightingale. Air-sea fluxes of oxygenated volatile organic compounds across the atlantic ocean. Atmospheric Chemistry and Physics, 14(14):7499-7517, 2014 . 